\title{
Convex Structures and Operational Quantum Mechanics
}

\author{
S. Gudder \\ Department of Mathematics, University of Denver, Denver, Colorado, USA
}

Received May 29, 1972; in revised form September 15, 1972

\begin{abstract}
A general mathematical framework called a convex structure is introduced. This framework generalizes the usual concept of a convex set in a real linear space. A metric is constructed on a convex structure and it is shown that mappings which preserve the structure are contractions. Convex structures which are isomomorphic to convex sets are characterized and for such convex structures it is shown that the metric is induced by a norm and that structure preserving mappings can be extended to bounded linear operators.

Convex structures are shown to give an axiomatization of the states of a physical system and the metric is physically motivated. We demonstrate how convex structures give a generalizing and unifying formalism for convex set and operational methods in axiomatic quantum mechanics.
\end{abstract}

\section{Introduction}

Until recently the main axiomatic frameworks for quantum mechanics have been the $C^{*}$-algebra approach $[6,14]$ and the "quantum logic" approach $[4,7,15]$. Recently a new and more general method of attack has been discussed, which might be called the "convex set" or "operational" approach. This method has been emphasized by Ludwig et al. [13] Gunson [5], Mielnik [9, 10], Davies and Lewis [2] and others [3,11]. In this framework a basic role is played by the convex set of normalized states $S_{1}$ together with the geometric properties of the boundary of $S_{1}$. Although the particular terminology, physical interpretations, and certain details of these investigations may differ, they all use convex set methods.

In this paper we introduce a framework which is even more general but which we feel forms a unification of the convex set or operational approaches and at the same time provides a new and perhaps useful mathematical tool for further investigations. In this framework the only primitive axiomatic elements are the normalized states $S_{1}$ of a physical system and the only operation postulated on $S_{1}$ is that of forming mixtures. In this way the convex structure of $S_{1}$ is isolated and is, in fact, the only structure of $S_{1}$. 


\section{Convex Structures}

Let $S_{1}=\{p, q, r, \ldots\}$ be the set of normalized states for some physical system. For generality we do not specify any particular form for these states but take them to be undefined, primitive elements. In different axiomatic models for quantum mechanics the states take various forms. In the conventional model, for example, the states are positive trace-class operators with trace one $[7,15]$; in the quantum logic approach, the states are probability measures on an orthomodular lattice $[4,7,15]$; in the algebraic approach, the states are positive, normal, linear functionals on a $C^{*}$-algebra $[6,14]$; in the operational approach, the states are positive elements of an ordered Banach space $[2,10]$. Although in these models the normalized states form a convex subset of a vector space we can formulate an axiomatic framework without any linear structure whatsoever. Thus our theory not only generalizes the usual models but leaves open the possibility of non-linear structures for quantum mechanics $[9,10]$.

We now assume there is a notion of mixtures of states. Thus if $\lambda_{1}, \lambda_{2} \geqq 0, \lambda_{1}+\lambda_{2}=1, p, q \in S_{1}$ we assume there is a state $T_{2}\left(\lambda_{1}, p ; \lambda_{2}, q\right)$ which may be interpreted as a mixture with $\lambda_{1}$ parts $p$ and $\lambda_{2}$ parts $q$. Depending upon ones interpretation $T_{2}\left(\lambda_{1}, p ; \lambda_{2}, q\right)$ may be looked upon as a state in which the system is in state $p$ with probability $\lambda_{1}$ or state $q$ with probability $\lambda_{2}$; or $T_{2}\left(\lambda_{1}, p ; \lambda_{2} . q\right)$ may describe a beam of non-interacting particles of two types in proportion $\lambda_{1}$ to $\lambda_{2}$; or some other interpretation. In any case it is clear that $T_{2}$ is a map $T_{2}:\left\{\left(\lambda_{1}, p ; \lambda_{2}, q\right): \lambda_{1}, \lambda_{2} \geqq 0, \lambda_{1}+\lambda_{2}=1, p, q \in S_{1}\right\} \rightarrow S_{1}$ satisfying:

(1) $T_{2}\left(\lambda_{1}, p ; \lambda_{2}, q\right)=T_{2}\left(\lambda_{2}, q ; \lambda_{1}, p\right)$

(2) $T_{2}(1, p ; 0, q)=p$.

Furthermore, we would like to form mix tures of three normalized states so we assume the existence of a map

$$
T_{3}:\left\{\left(\lambda_{1}, p_{1} ; \lambda_{2}, p_{2} ; \lambda_{3}, p_{3}\right): \lambda_{i} \geqq 0, \sum_{1}^{3} \lambda_{i}=1, p_{1}, p_{2}, p_{3} \in S_{1}\right\} \rightarrow S_{1}
$$

satisfying:

$$
T_{3}\left(\lambda_{i}, p_{i} ; \lambda_{j}, p_{j} ; \lambda_{k}, p_{k}\right)=T_{3}\left(\lambda_{1}, p_{1} ; \lambda_{2}, p_{2} ; \lambda_{3}, p_{3}\right)
$$

whenever $(i, j, k)$ is a permutation of $(1,2,3)$,

(4) if $\lambda_{1} \neq 1$ then

$$
\begin{aligned}
& T_{3}\left(\lambda_{1}, p_{1} ; \lambda_{2}, p_{2} ; \lambda_{3}, p_{3}\right) \\
& \quad=T_{2}\left(\lambda_{1}, p_{1} ; 1-\lambda_{1}, T_{2}\left(\lambda_{2}\left(1-\lambda_{1}\right)^{-1}, p_{2} ; \lambda_{3}\left(1-\lambda_{1}\right)^{-1}, p_{3}\right)\right) .
\end{aligned}
$$


Condition (3) is clear, while condition (4) gives the connection between $T_{3}$ and $T_{2}$ and follows from the fact that the right-hand side is a mixture of $\lambda_{1}$ parts $p_{1}$ and $1-\lambda_{1}$ parts a state with $\lambda_{2}\left(1-\lambda_{1}\right)^{-1}$ parts $p_{2}$ and $\lambda_{3}\left(1-\lambda_{1}\right)^{-1}$ parts $p_{3}$ so the resulting state has $\lambda_{1}$ parts $p_{1}, \lambda_{2}$ parts $p_{2}$ and $\lambda_{3}$ parts $p_{3}$.

A set $S_{1}$ with two maps $T_{2}, T_{3}$ satisfying conditions (1)-(4) is called a convex structure. Let us define the map $T:[0,1] \times S_{1} \times S_{1} \rightarrow S_{1}$ by $T(\lambda, p, q) \equiv\langle\lambda, p, q\rangle=T_{2}(1-\lambda, p ; \lambda, q)$. Of course, $\langle\lambda, p, q\rangle$ represents a mixture with $1-\lambda$ parts $p$ and $\lambda$ parts $q$. The reason for defining $\langle\lambda, p, q\rangle$ as $T_{2}(1-\lambda, p ; \lambda, q)$ instead of $T_{2}(\lambda, p ; 1-\lambda, q)$ is merely for the convenience of making our later formulas simpler and also to agree with the standard notation in convex sets.

Theorem 2.1. If $\left(S_{1}, T_{2}, T_{3}\right)$ is a convex structure then $T(\lambda, p, q)$ $\equiv\langle\lambda, p, q\rangle=T_{2}(1-\lambda, p ; \lambda, q)$ satisfies:

(a) $\langle\lambda, p, q\rangle=\langle 1-\lambda, q, p\rangle$ (commutativity)

(b) $\langle 0, p, q\rangle=p$ (endpoint condition)

(c) $\langle\lambda, p,\langle\mu, q, r\rangle\rangle=\left\langle\lambda \mu,\left\langle\lambda(1-\mu)(1-\lambda \mu)^{-1}, p, q\right\rangle, r\right\rangle(\lambda \mu \neq 1)$ (associativity).

Conversely, let $S_{1}=\{p, q, r, \ldots\}$ be a set and let $T:[0,1] \times S_{1} \times S_{1} \rightarrow S_{1}$, $T(\lambda, p, q) \equiv\langle\lambda, p, q\rangle$ satisfy (a), (b) and (c). If we define

and

$$
T_{2}:\left\{\left(\lambda_{1}, p ; \lambda_{2}, q\right): \lambda_{1}, \lambda_{2} \geqq 0, \lambda_{1}+\lambda_{2}=1, p, q \in S_{1}\right\} \rightarrow S_{1}
$$

$$
T_{3}:\left\{\left(\lambda_{1}, p_{1} ; \lambda_{2}, p_{2} ; \lambda_{3}, p_{3}\right): \lambda_{i} \geqq 0, \sum_{1}^{3} \lambda_{i}=1, p_{1}, p_{2}, p_{3} \in S_{1}\right\} \rightarrow S_{1}
$$

by $T_{2}\left(\lambda_{1}, p ; \lambda_{2}, q\right)=\left\langle 1-\lambda_{1}, p, q\right\rangle$ and

$$
T_{3}\left(\lambda_{1}, p ; \lambda_{2}, q ; \lambda_{3}, r\right)=\left\langle 1-\lambda_{1}, p ;\left\langle\lambda_{3}\left(1-\lambda_{1}\right)^{-1}, q, r\right\rangle\right\rangle
$$

if $\lambda_{1} \neq 1,=p$ if $\lambda_{1}=1$, then $\left(S_{1}, T_{2}, T_{3}\right)$ is a convex structure.

Proof. (a) $\langle\lambda, p, q\rangle=T_{2}(1-\lambda, p ; \lambda, q)=T_{2}(\lambda, q ; 1-\lambda, p)=\langle 1-\lambda, q, p\rangle$.

(b) $\langle 0, p, q\rangle=T_{2}(1, p ; 0, q)=p$.

(c) $\langle\lambda, p,\langle\mu, q, r\rangle\rangle=T_{2}(1-\lambda, p ; \lambda,\langle\mu, q, r\rangle)$

$$
\begin{aligned}
= & T_{2}\left(1-\lambda, p ; \lambda, T_{2}(1-\mu, q ; \mu, r)\right) \\
= & T_{3}(1-\lambda, p ;(1-\mu) \lambda, q ; \mu \lambda, r) \\
= & T_{3}(\mu \lambda, r ; 1-\lambda, p ;(1-\mu) \lambda, q) \\
= & T_{2}\left(\mu \lambda, r ; 1-\mu \lambda, T_{2}\left((1-\lambda)(1-\mu \lambda)^{-1}, p ;\right.\right. \\
& \left.\left.\cdot(1-\mu) \lambda(1-\mu \lambda)^{-1}, q\right)\right) \\
= & \left\langle 1-\mu \lambda, r,\left\langle\lambda(1-\mu)(1-\mu \lambda)^{-1}, p, q\right\rangle\right\rangle \\
= & \left\langle\mu \lambda,\left\langle\lambda(1-\mu)(1-\mu \lambda)^{-1}, p, q\right\rangle, r\right\rangle .
\end{aligned}
$$


(1) $T_{2}\left(\lambda_{1}, p_{1} ; \lambda_{2}, q\right)=\left\langle 1-\lambda_{1}, p, q\right\rangle=\left\langle\lambda_{1}, q, p\right\rangle=\left\langle 1-\lambda_{2}, q, p\right\rangle$

$$
=T_{2}\left(\lambda_{2}, q ; \lambda_{1}, p\right) \text {. }
$$

(2) $T_{2}(1, p, 0, q)=\langle 0, p, q\rangle=p$.

(3) $T_{3}\left(\lambda_{1}, p_{1} ; \lambda_{2}, p_{2} ; \lambda_{3}, p_{3}\right)=\left\langle 1-\lambda_{1}, p_{1},\left\langle\lambda_{3}\left(1-\lambda_{1}\right)^{-1}, p_{2}, p_{3}\right\rangle\right\rangle$

$$
\begin{aligned}
& =\left\langle\lambda_{3},\left\langle\lambda_{2}\left(1-\lambda_{3}\right)^{-1}, p_{1}, p_{2}\right\rangle, p_{3}\right\rangle \\
& =\left\langle 1-\lambda_{3}, p_{3},\left\langle\lambda_{2}\left(1-\lambda_{3}\right)^{-1}, p_{1}, p_{2}\right\rangle\right\rangle \\
& =T_{3}\left(\lambda_{3}, p_{3} ; \lambda_{1}, p_{1} ; \lambda_{2}, p_{2}\right)
\end{aligned}
$$

and the other permutations are similar.

(4) $\lambda_{1} \neq 1$ then,

$$
\begin{aligned}
T_{3}\left(\lambda_{1}, p_{1} ; \lambda_{2}, p_{2} ; \lambda_{3}, p_{3}\right)= & \left\langle 1-\lambda_{1}, p_{1},\left\langle\lambda_{3}\left(1-\lambda_{1}\right)^{-1}, p_{2}, p_{3}\right\rangle\right\rangle \\
= & T_{2}\left(\lambda_{1}, p_{1} ;\left(1-\lambda_{1}\right),\left\langle\lambda_{3}\left(1-\lambda_{1}\right)^{-1}, p_{2}, p_{3}\right\rangle\right) \\
= & T_{2}\left(\lambda_{1}, p_{1} ;\left(1-\lambda_{1}\right), T_{2}\left(\lambda_{2}\left(1-\lambda_{1}\right)^{-1}, p_{2} ;\right.\right. \\
& \left.\left.\lambda_{3}\left(1-\lambda_{1}\right)^{-1}, p_{3}\right)\right) .
\end{aligned}
$$

Thus an equivalent definition of a convex structure is a pair $\left(S_{1}, T\right)$

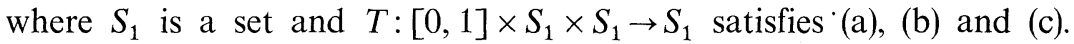
Although our first definition is more physically motivated, the second is more convenient mathematically so we will use the second in the sequel. An example of a convex structure is a convex set $S_{0}$ in a real linear space where $T:[0,1] \times S_{0} \times S_{0} \rightarrow S_{0}$ is $T(\lambda, p, q)=(1-\lambda) p+\lambda q$. However one can construct convex structures that are not of this form. For instance, there are simple examples [1] of convex structures $S_{1}$ with finitely many elements and since a non-empty, non-singleton convex set $S_{0}$ must have infinitely many elements, $S_{1}$ cannot be isomorphic to an $S_{0}$. Our next result will characterize those convex structures that are isomorphic to a convex set.

A convex prestructure is a set $S=\{p, q, r, \ldots\}$ together with a map $T:[0,1] \times S \times S \rightarrow S$ denoted by $T(\lambda, p, q)=\langle\lambda, p, q\rangle$. Of course, any set is a convex prestructure since we have placed no requirements on $T$. If $S_{1}, S_{2}$ are convex prestructures, a map $A: S_{1} \rightarrow S_{2}$ is affine if $\left.A<\lambda, p, q\right\rangle_{1}$ $=\langle\lambda, A p, A q\rangle_{2}$ for every $\lambda \in[0,1], p, q \in S_{1}$. We say $S_{1}$ and $S_{2}$ are isomorphic if there is an affine bijection from $S_{1}$ to $S_{2}$. If $S_{0}$ is a convex subset of a real vector space we always assume $S_{0}$ is equipped with its usual convex structure. An affine functional is an affine map $f$ from a convex prestructure $S$ to the real line $R$; that is, $f(\langle\lambda, p, q\rangle)=(1-\lambda) f(p)$ $+\lambda f(q)$ for all $\lambda \in[0,1], p, q \in S$. We let $S^{*}$ denote the set of all affine functionals on $S$ and say that $S^{*}$ is total if for $p \neq q \in S$ there is an $f \in S^{*}$ such that $f(p) \neq f(q)$.

Theorem 2.2. A convex prestructure $S$ is isomorphic to a convex set if and only if $S^{*}$ is total. 
Proof. Suppose $S_{0}$ is a convex set and $F: S \rightarrow S_{0}$ is an isomorphism. If $S_{0}$ is a convex subset of the vector space $V$, it is well-known that the algebraic dual $V^{*}$ is total over $V$. Restricting the elements of $V^{*}$ to $S_{0}$ we get a total set of affine functionals for $S_{0}$. Now if $f \in V^{*}$ then $f \circ F \in S^{*}$ so $S^{*}$ is total. Conversely, suppose $S^{*}$ is total. For $p \in S$ define $J(p): S^{*} \rightarrow R$ by $J(p) f=f(p)$. Clearly $S^{*}$ is a vector space under pointwise operations and $J(p) \in S^{* *}$ so that $J(S) \subseteq S^{* *}$. Now $J(S)$ is a convex set since for $J(p), J(q) \in J(S)$ and $\lambda \in[0,1]$ we have for $f \in S^{*}$,

$$
[(1-\lambda) J(p)+\lambda J(q)] f=(1-\lambda) f(p)+\lambda f(q)=f(\langle\lambda, p, q\rangle)=J(\langle\lambda, p, q\rangle) f
$$

so $(1-\lambda) J(p)+\lambda J(q)=J(\langle\lambda, p, q\rangle) \in J(S)$. Now $J: S \rightarrow S^{* *}$ is injective if and only if $S^{*}$ is total. Indeed, if $S^{*}$ is total and $p \neq q \in S$ then there is an $f \in S^{*}$ such that $f(p) \neq f(q)$ so $J(p) \neq J(q)$ and conversely, if $J$ is injective and $p \neq q \in S$ then $J(p) \neq J(q)$ so there is an $f \in S^{*}$ such that $f(p)=J(p) f$ $\neq J(q) f=f(q)$. It follows that $J: S \rightarrow J(S)$ is an isomorphism.

We would now like to define a distance between normalized states $p, q$. The closeness of $p$ to $q$ can be measured by comparing mixtures $\left\langle\lambda, p, p_{1}\right\rangle,\left\langle\lambda, q, q_{1}\right\rangle$ of $p$ and $q$ with other normalizes states. If $p$ and $q$ are very close one would expect to find a mixture containing mostly $p$ equal to a mixture containing mostly $q$; that is, $\left\langle\lambda, p, p_{1}\right\rangle=\left\langle\lambda, q, q_{1}\right\rangle$ in which $\lambda$ is very small. Conversely, if $\left\langle\lambda, p, p_{1}\right\rangle=\left\langle\lambda, q, q_{1}\right\rangle$ and $\lambda$ is small one expects that $p$ and $q$ are close. Thus the parameters $\lambda$ such that $\left\langle\lambda, p, p_{1}\right\rangle=\left\langle\lambda, q, q_{1}\right\rangle$ give a measure of the closeness of $p$ to $q$. We thus define

$$
\sigma(p, q)=\inf \left\{0 \leqq \lambda \leqq 1:\left\langle\lambda, p, p_{1}\right\rangle=\left\langle\lambda, q, q_{1}\right\rangle, p_{1}, q_{1} \in S_{1}\right\},
$$

where $S_{1}$ is a convex structure. Notice that since $\left\langle 2^{-1}, p, q\right\rangle=\left\langle 2^{-1}, q, p\right\rangle$. we have $0 \leqq \sigma(p, q) \leqq \frac{1}{2}$. It turns out to be more useful to make a change of scale and define the distance between $p$ and $q$ to be $\varrho(p, q)=\sigma(p, q)$ $\cdot[1-\sigma(p, q)]^{-1}$ so that $0 \leqq \varrho(p, q) \leqq 1$. I am indebted to W. Cornette for help with the following proof.

Theorem 2.3. On a convex structure $S_{1}, \sigma$ and $\varrho$ are semimetrics.

Proof. It is clear that $\sigma$ and $\varrho$ are non-negative and symmetric. Since $\left\langle 0, p, q_{1}\right\rangle=\left\langle 0, p, q_{1}\right\rangle$ for every $p_{1}, q_{1} \in S_{1}$ we have $\sigma(p, p)=\varrho(p, p)=0$. We now prove the triangle inequality. Clearly, $\sigma(p, q) \leqq \sigma(p, s)+\sigma(s, q)$ if $p=s$ or $q=s$ so we can exclude these cases. Suppose

$$
\lambda_{1} \in\left\{0<\lambda<1:\left\langle\lambda, p, p_{1}\right\rangle=\left\langle\lambda, s, s_{1}\right\rangle, p_{1}, s_{1} \in S_{1}\right\}
$$

and $\lambda_{2} \in\left\{0<\lambda<1:\left\langle\lambda, s, s_{2}\right\rangle=\left\langle\lambda, q, q_{1}\right\rangle, s_{2}, q_{1} \in S_{1}\right\}$. Letting $\lambda_{3}=\lambda_{1}+\lambda_{2}$ $-2 \lambda_{1} \lambda_{2}, \quad p_{2}=\left\langle\lambda_{2}\left(1-\lambda_{1}\right) \lambda_{3}^{-1}, p_{1}, s_{2}\right\rangle, \quad q_{2}=\left\langle\lambda_{2}\left(1-\lambda_{1}\right) \lambda_{3}^{-1}, s_{1} q_{1}\right\rangle$ and 
$\lambda_{0}=\lambda_{3}\left(1-\lambda_{1} \lambda_{2}\right)^{-1}$ we have:

$$
\begin{aligned}
\left\langle\lambda_{0}, p, p_{2}\right\rangle & =\left\langle\lambda_{3}\left(1-\lambda_{1} \lambda_{2}\right)^{-1}, p,\left\langle\lambda_{2}\left(1-\lambda_{1}\right) \lambda_{3}^{-1}, p_{1}, s_{2}\right\rangle\right\rangle \\
& =\left\langle\lambda_{2}\left(1-\lambda_{1}\right)\left(1-\lambda_{1} \lambda_{2}\right)^{-1},\left\langle\lambda_{1}, p, p_{1}\right\rangle, s_{2}\right\rangle \\
& =\left\langle\lambda_{2}\left(1-\lambda_{1}\right)\left(1-\lambda_{1} \lambda_{2}\right)^{-1},\left\langle\lambda_{1}, s, s_{1}\right\rangle, s_{2}\right\rangle \\
& =\left\langle\left(1-\lambda_{2}\right)\left(1-\lambda_{1} \lambda_{2}\right)^{-1}, s_{2},\left\langle\lambda_{1}, s, s_{1}\right\rangle\right\rangle \\
& =\left\langle\lambda_{1}\left(1-\lambda_{2}\right)\left(1-\lambda_{1} \lambda_{2}\right)^{-1},\left\langle 1-\lambda_{2}, s_{2}, s\right\rangle, s_{1}\right\rangle \\
& =\left\langle\left(1-\lambda_{1}\right)\left(1-\lambda_{1} \lambda_{2}\right)^{-1}, s_{1},\left\langle\lambda_{2}, q, q_{1}\right\rangle\right\rangle \\
& =\left\langle\left(1-\lambda_{1}\right)\left(1-\lambda_{2}\right)\left(1-\lambda_{1} \lambda_{2}\right)^{-1},\left\langle\lambda_{2}\left(1-\lambda_{1}\right) \lambda_{3}^{-1}, s_{1}, q_{1}\right\rangle, q\right\rangle \\
& =\left\langle\lambda_{3}\left(1-\lambda_{1} \lambda_{2}\right)^{-1}, q, q_{2}\right\rangle=\left\langle\lambda_{0}, q, q_{2}\right\rangle
\end{aligned}
$$

so $\lambda_{0} \in\left\{0<\lambda<1:\left\langle\lambda, p, p_{2}\right\rangle=\left\langle\lambda, q, q_{2}\right\rangle, p_{2}, q_{2} \in S_{1}\right\}$. Now since $\lambda_{0}\left(1-\lambda_{0}\right)^{-1}=\lambda_{1}\left(1-\lambda_{1}\right)^{-1}+\lambda_{2}\left(1-\lambda_{2}\right)^{-1}$ we have

$$
\begin{aligned}
\varrho(p, q)= & \sigma(p, q)[1-\sigma(p, q)]^{-1} \leqq \sigma(p, s)[1-\sigma(p, s)]^{-1} \\
& +\sigma(s, q)\left[(1-\sigma(s, q)]^{-1}=\varrho(p, s)+\varrho(s, q)\right.
\end{aligned}
$$

The triangle inequality for $\sigma$ follows in a similar way using the fact that $\lambda_{0} \leqq \lambda_{1}+\lambda_{2}$.

We call $\varrho$ the intrinsic semimetric for $S_{1}$. As an example, let $p<q \in R$ and let us compute $\varrho(p, q)$ relative to some bounded convex subset $S_{0}$ of $R$. Since the only convex subsets of $R$ are intervals we may assume that $S_{0}$ is a closed interval $\left[p_{0}, q_{0}\right], p_{0}<q_{0}, p, q \in\left[p_{0}, q_{0}\right]$. It is easy to see that relative to $S_{0}, \sigma(p, q)=(p-q)\left[(p-q)+\left(p_{0}-q_{0}\right)\right]^{-1}$ and $\varrho(p, q)=(p-q)\left(p_{0}-q_{0}\right)^{-1}$. Thus $\varrho$ is a metric on $\left[p_{0}, q_{0}\right]$ and is equivalent to the Euclidean metric. Notice however, that if $\varrho$ is computed relative to an unbounded convex set, then all distances are zero and $\varrho$ is the trivial semimetric $\varrho \equiv 0$.

Lemma 2.4. A necessary and sufficient condition for $\sigma$, @ to be metrics is that whenever there are sequences $\lambda_{i} \in[0,1], p_{i}, q_{i} \in S_{1}$ which satisfy $\lim _{i \rightarrow \infty} \lambda_{i}=0,\left\langle\lambda_{i}, p, p_{i}\right\rangle=\left\langle\lambda_{i}, q, q_{i}\right\rangle$ then $p=q$.

Proof. Clearly $\varrho$ is a metric if and only if $\sigma$ is. Now if $\sigma$ is a metric, since $\sigma(p, q) \leqq \lambda_{i}$ for all $i$ we have $p=q$. Conversely, if $\sigma(p, q)=0$ then $V=\left\{0 \leqq \lambda \leqq 1:\left\langle\lambda, p, p_{1}\right\rangle=\left\langle\lambda, q, q_{1}\right\rangle, p_{1}, q_{1} \in S_{1}\right\}$ either contains 0 or has 0 as a limit point. In the former case $p=\left\langle 0, p, p_{1}\right\rangle=\left\langle 0, q, q_{1}\right\rangle=q$. In the latter case there exist $\lambda_{i} \in V$ with $\lim \lambda_{i}=0$ so again $p=q$.

Corollary 2.5. Let $S_{0}$ be a convex set in a real vector space $X$. If there is a topology on $X$ that makes $X$ a Hausdorff topological vector space in which $S_{0}$ is bounded, then $\varrho$ is a metric. 
Proof. Suppose there are sequences $\lambda_{i} \in[0,1], \lim \lambda_{i}=0, p_{i}, q_{i} \in S_{0}$ such that $\left(1-\lambda_{i}\right) p+\lambda_{i} p_{i}=\left(1-\lambda_{i}\right) q+\lambda_{i} q_{i}$. Then $p-q=\lambda_{i}(p-q)$ $+\lambda_{i}\left(q_{i}-p_{i}\right)$. Let $\Lambda$ be any neighborhood of 0 . Then there is a neighborhood $W$ of 0 such that $W+W+W \leqq \Lambda$. Now for $i$ sufficiently large $\lambda_{i}(p-q) \in W$. Since $S_{0}$ is bounded there is a $\mu>0$ such that $\lambda S_{0} \leqq W$ for $|\lambda| \leqq \mu$. Thus for $i$ sufficiently large $\lambda_{i} q_{i}-\lambda_{i} p_{i} \in W+W$. Hence for sufficiently large $i$, $p-q \in W+W+W \leqq \Lambda$. Since $X$ is Hausdorff, $p-q=0$ and $p=q$.

The converse of the above corollary holds for finite dimensional spaces $X$. Indeed if $S_{0}$ is unbounded in $X$ then one can show there is an infinite ray in $S_{0}$ and hence any two points on this ray will have distance zero. The converse of the corollary need not hold in infinite dimensional spaces.

If $S_{1}, S_{2}$ are convex structures we denote the affine maps $A: S_{1} \rightarrow S_{2}$ by $A f\left(S_{1}, S_{2}\right)$. We use the notation $A f\left(S_{1}\right)$ for $A f\left(S_{1}, S_{1}\right)$ and the group of bijections in $A f\left(S_{1}\right)$ is denoted $\operatorname{Aut}\left(S_{1}\right)$.

Lemma 2.6. (1) If $A \in A f\left(S_{1}, S_{2}\right)$ then $A$ is a contraction (i.e. $\left.\varrho_{2}(A p, A q) \leqq \varrho_{1}(p, q)\right)$. (2) If $A \in A f\left(S_{1}, S_{2}\right)$ is bijective then $A$ is an isometry.

$$
\begin{aligned}
\text { Proof. (1) } \sigma_{2}(A p, A q)= & \inf \left\{0 \leqq \lambda \leqq 1:\left\langle\lambda, A p, p_{1}\right\rangle_{2}\right. \\
& \left.=\left\langle\lambda, A q, q_{1}\right\rangle_{2}, p_{1}, q_{1} \in S_{2}\right\} \\
\leqq & \inf \left\{0 \leqq \lambda \leqq 1: A<\lambda, p, q_{1}\right\rangle_{1} \\
& \left.=A\left\langle\lambda, q, q_{1}\right\rangle_{1}, p_{1}, q_{1} \in S_{1}\right\} \\
\leqq & \inf \left\{0 \leqq \lambda \leqq 1:\left\langle\lambda, p, p_{1}\right\rangle_{1}\right. \\
= & \left.\sigma_{1}(p, q) . \quad=\left\langle\lambda, q, q_{1}\right\rangle_{1}, p_{1}, q_{1} \in S_{1}\right\}
\end{aligned}
$$

Since $x(1-x)^{-1}$ is monotone increasing function we have $\varrho_{2}(A p, A q)$ $\leqq \varrho_{1}(p, q)$. (2) follows from (1).

It follows, of course, that any $A \in A f\left(S_{1}, S_{2}\right)$ is continuous relative to the intrinsic metrics. As an application, if $f \in S_{1}^{*}$ and $0 \leqq f \leqq 1$ then $|f(p)-f(q)| \leqq \varrho(p, q)$.

Let $S_{1}$ be a convex structure. For applications it is important to consider the set $S=S_{1}^{+}=\left\{(\alpha, p): p \in S_{1}, \alpha \geqq 0\right\}$. We define $(\alpha, p)=(\beta, q)$ if $\alpha=\beta \neq 0$ and $p=\mathrm{q}$, and $(0, p)=(0, q) \equiv 0$ for all $p, q \in S_{1}$. For convenience we write $\alpha p$ instead of $(\alpha, p)$. If $S_{1}$ corresponds to the normalized states of a physical system, then $S$ corresponds to the states. For $\alpha p \in S$ and $\beta \geqq 0$ we define $\beta(\alpha p)=(\beta \alpha) p$, and clearly $\beta(\alpha p)=\alpha(\beta p)$. For $\alpha p, \beta q \in S$, $\lambda \in[0,1]$, we define $\langle\lambda, \alpha p, \beta q\rangle=0$ if $(1-\lambda) \alpha+\lambda \beta=0$ and otherwise $\langle\lambda, \alpha p, \beta q\rangle=[(1-\lambda) \alpha+\lambda \beta]\left\langle\lambda \beta[(1-\lambda) \alpha+\lambda \beta]^{-1}, p, q\right\rangle$.

Lemma 2.7. $(S,\langle\cdot, \cdot, \cdot\rangle)$ is a convex structure. 
Proof. (a) Let $\gamma=(1-\lambda) \alpha+\lambda \beta$. Then for $\gamma \neq 0$

$$
\begin{aligned}
\langle\lambda, \alpha p, \beta q\rangle & =\gamma\left\langle\lambda \beta \gamma^{-1}, p, q\right\rangle=\gamma\left\langle 1-\lambda \beta \gamma^{-1}, q, p\right\rangle=\gamma\left\langle(1-\lambda) \alpha \gamma^{-1}, q, p\right\rangle \\
& =\langle 1-\lambda, \beta q, \alpha p\rangle .
\end{aligned}
$$

If $\gamma=0$ the equality clearly holds. (b) $\langle 0, \alpha p, \beta q\rangle=\alpha\langle 0, p, q\rangle=\alpha p$. (c) Letting $\xi=(1-\mu) \beta+\mu \delta, \tau=(1-\lambda) \alpha+\lambda \xi$, and $\sigma=\alpha(1-\lambda)(1-\lambda \mu)^{-1}$ $+\beta \lambda(1-\mu)(1-\lambda \mu)^{-1}$ we have

$$
\begin{aligned}
\langle\lambda, \alpha p,\langle\mu, \beta q, \delta r\rangle\rangle & =\left\langle\lambda, \alpha p, \xi\left\langle\mu \delta \xi^{-1}, q, r\right\rangle\right\rangle \\
& =\tau\left\langle\lambda \xi \tau^{-1}, p,\left\langle\mu \delta \xi^{-1}, q, r\right\rangle\right\rangle \\
& =\tau\left\langle\lambda \mu \delta \tau^{-1},\left\langle\lambda \xi \tau^{-1}\left(1-\mu \delta \xi^{-1}\right)\left(1-\lambda \mu \delta \tau^{-1}\right)^{-1},\right.\right. \\
& \quad p, q\rangle, r\rangle \\
& =\tau\left\langle\lambda \mu \delta \tau^{-1},\left\langle\lambda(1-\mu) \beta(1-\lambda \mu)^{-1} \sigma^{-1}, p, q\right\rangle, r\right\rangle \\
& =\left\langle\lambda \mu, \sigma\left\langle\lambda(1-\mu) \beta(1-\lambda \mu)^{-1} \sigma^{-1}, p, q\right\rangle, \delta r\right\rangle \\
& =\left\langle\lambda \mu,\left\langle\lambda(1-\mu)(1-\lambda \mu)^{-1}, \alpha p, \beta q\right\rangle, \delta r\right\rangle .
\end{aligned}
$$

Thus all our results for convex structures can be applied to $S$. In particular $S$ has an intrinsic metric

Let $S_{3}$ be a convex structure. We say $S_{2}$ is a convex substructure of $S_{3}$ if $S_{2} \subseteq S_{3}$ and $\langle\lambda, p, q\rangle \in S_{2}$ for all $\lambda \in[0,1], p, q \in S_{2}$. If we identify elements of $S_{1}$ with elements of $S_{1}^{+}$of the form $1 \cdot p, p \in S_{1}$ then $S_{1}$ is a convex substructure of $S_{1}^{+}$. We now show how to extend elements of $S_{1}^{*}$ to elements of $S_{1}^{+*}$.

Lemma 2.8. (a) If $g \in S_{1}^{+*}$ then $g(\alpha p)=\alpha g(p)+(1-\alpha) g(0)$ for all $\alpha \geqq 0, p \in S_{1}$. (b) If $f \in S_{1}^{*}$ and $c \in R$ there exists a unique extension $g \in S_{1}^{+*}$ of $f$ satisfying $g(0)=c$; in fact, $g(\alpha p)=\alpha f(p)+(1-\alpha) c$.

Proof. (a) For any $\lambda \in[0,1], \beta \geqq 0, p \in S_{1}$ we have $\langle\lambda, 0 p, \beta p\rangle$ $=\lambda \beta\langle 1, p, p\rangle=\lambda \beta p$ and hence $g(\lambda \beta p)=(1-\lambda) g(0)+\lambda g(\beta p)$. If $0 \leqq \alpha \leqq 1$ let $\beta=1$ and $\lambda=\alpha$ to obtain the result. If $\alpha>1$ let $\beta=\alpha$ and $\lambda=\alpha^{-1}$ to obtain $g(p)=\left(1-\alpha^{-1}\right) g(0)+\alpha^{-1} g(\alpha p)$ which again gives the result. (b) Defining $g(\alpha p)=\alpha f(p)+(1-\alpha) c$ we need only show $g \in S_{1}^{+*}$ since clearly $g$ is an extension of $f, g(0)=c$ and uniqueness follows from (a). Now letting $\gamma=(1-\lambda) \alpha+\lambda \beta$,

$$
\begin{aligned}
g(\langle\lambda, \alpha p, \beta q\rangle) & \left.=g\left(\gamma<\lambda \beta \gamma^{-1}, p, q\right\rangle\right) \\
& =\gamma f\left(\left\langle\lambda \beta \gamma^{-1}, p, q\right\rangle\right)+(1-\gamma) c \\
& =\gamma\left[\left(1-\lambda \beta \gamma^{-1}\right) f(p)+\lambda \beta \gamma^{-1} f(g)\right]+(1-\gamma) c \\
& =(1-\lambda) \alpha f(p)+\lambda \beta f(g)+(1-\gamma) c \\
& =(1-\lambda)[\alpha f(p)+(1-\alpha) c]+\lambda[\beta f(g)+(1-\beta) c] \\
& =(1-\lambda) g(\alpha p)+\lambda g(\beta q) .
\end{aligned}
$$

This completes the proof. 
If $f_{1} \in S_{1}^{*}$ is defined by $f_{1}(p)=1$ for all $p \in S_{1}$ its extension $\tau \in S_{1}^{+*}$ given by $\tau(\alpha p)=\alpha$ will be quite important in the applications.

\section{The Induced Seminorm}

We now investigate the intrinsic semimetric in the special case of convex sets. In this section $S_{0}$ will denote a convex set in a real vector space $V$ and $\varrho$ the intrinsic semimetric on $S_{0}$. We first recall some definitions. $S_{0}$ is absorbing if for any $x \in V$ there is a $\delta(x)>0$ such that $\lambda x \in S_{0}$ for any $\lambda$ with $|\lambda| \leqq \delta(x)$. $S_{0}$ is balanced if $\lambda x \in S_{0}$ for any $x \in S_{0}$, $|\lambda| \leqq 1 . S_{0}$ is radial if $x \in S_{0}$ implies $\lambda x \in S_{0}$ for any $0 \leqq \lambda \leqq 1 . S_{0}$ is normalized if $x \in S_{0}$ implies $\alpha x \notin S_{0}$ for any $\alpha \neq 1$. If $K=\left\{\alpha S_{0}: \alpha \geqq 0\right\}$ then $K$ is wedge in $V$, i.e., $K+K \leqq K$ and $\alpha K \subseteq K$ for any $\alpha \geqq 0$. We let $X=K-K \leqq V$ be the subspace of $V$ generated by $K$.

Let $D=\left\{c p-d q: 0 \leqq c, d \leqq 1 ; p, q \in S_{0}\right\}$. Then $D$ is a convex, balanced, absorbing subset of $X$ containing 0 . Notice $D=S_{0}-S_{0}$ if $S_{0}$ is radial. For $x \in X$ let $|x|=\inf \{\lambda>0: x \in \lambda D\}$. Then $|\cdot|$ is the Minkowski functional [12] for $D$ in $X$. It is well-known that $|\cdot|$ is a seminorm and we call it the seminorm induced by the intrinsic semimetric $\varrho$. The reason for this terminology will become apparent from Theorem 3.2. We first give another way of computing $|\cdot|$ which is frequently more convenient.

Lemma 3.1. $|x|=\inf \left\{\max (c, d): x=c p-d q ; c, d \geqq 0 ; p, q \in S_{0}\right\}$.

Proof. By definition,

$$
|x|=\inf \left\{\lambda>0: x=\lambda c p-\lambda d q ; 0 \leqq c, d \leqq 1 ; p, q \in S_{0}\right\} .
$$

Now if $x=\lambda c p-\lambda d q, \quad 0 \leqq c, d \leqq 1$, then $x=(\lambda c) p-(\lambda d) q$ where $\max (\lambda c, \lambda d) \leqq \lambda$ so $\inf \left\{\max (c, d): x=c p-d q ; c, d \geqq 0 ; p, q \in S_{0}\right\} \leqq|x|$. Conversely, if $x=c p-d q, c, d>0, p, q \in S_{0}$ then

$$
x=\max (c, d)\left[c \max (c, d)^{-1}\right] p-\max (c, d)\left[d \max (c, d)^{-1}\right] q
$$

so the opposite inequality holds.

Theorem 3.2. If $S_{0}$ is normalized or radial then $|p-q|=\varrho(p, q)$ for all $p, q \in S_{0},|\cdot|$ is a norm if and only if $\varrho$ is a metric.

Proof. Let us first assume $S_{0}$ is normalized and $p, q \in S_{0}$. If $p-q=c p_{1}-d q_{1}, c, d \geqq 0, p_{1}, q_{1} \in S_{0}$ then $p+d q_{1}=q+c p_{1}$ so

$$
\begin{aligned}
(1+d)\left[(1+d)^{-1} p\right. & \left.+d(1+d)^{-1} q_{1}\right] \\
& =(1+c)\left[1(1+c)^{-1} q+c(1+c)^{-1} p_{1}\right] .
\end{aligned}
$$

Now since $q_{2} \equiv(1+c)^{-1} q+c(1+c)^{-1} p_{1} \in S_{0}$ and $(1+c)(1+d)^{-1} q_{2} \in S_{0}$ we must have $(1+c)(1+d)^{-1}=1$ so $c=d$. Thus all the representations of 
$p-q$ are of the form $p-q=c\left(p_{1}-q_{1}\right)$ for $c \geqq 0, p_{1}, q_{1} \in S_{0}$.

$$
\begin{aligned}
\sigma(p, q) & =\inf \left\{0 \leqq \lambda<1:(1-\lambda) p+\lambda p_{1}=(1-\lambda) q+\lambda q_{1}, p_{1}, q_{1} \in S_{0}\right\} \\
& =\inf \left\{0 \leqq \lambda<1: p-q=\lambda(1-\lambda)^{-1}\left(q_{1}-p_{1}\right), q_{1}, p_{1} \in S_{0}\right\} \\
& =\inf \left\{c(c+1)^{-1}, c \geqq 0: p-q=c\left(q_{1}-p_{1}\right), q_{1}, p_{1} \in S_{0}\right\} \\
& =\inf \left\{c \geqq 0: p-q=c\left(q_{1}-p_{1}\right)\right\}\left[\inf \left\{c \geqq 0: p-q=c\left(q_{1}-p_{1}\right)\right\}+1\right]^{-1} \\
& =|p-q|[|p-q|+1]^{-1} .
\end{aligned}
$$

Hence $\varrho(p, q)=\sigma(p, q)[1-\sigma(p, q)]^{-1}=|p-q|$. Now let us assume that $S_{0}$ is radial. Then suppose $p-q=c p_{1}-d q_{1}, p, q, p_{1}, q_{1} \in S_{0}$ where $c>d$. Then $p-q=c p_{1}-c(d / c) q_{1}$ where $(d / c) q_{1} \in S_{0}$. Thus for any representation of the form $p-q=c p_{1}-d q_{1}$ we have another representation $p-q=b\left(p_{2}-q_{2}\right)$ where $b=\max (c, d), p_{2}, q_{2} \in S_{0}$. We then obtain $|p-q|=\inf \left\{c \geqq 0: p-q=c\left(p_{1}-q_{1}\right), p_{1}, q_{1} \in S_{0}\right\}$ and just as in the normalized case we obtain $\varrho(p, q)=|p-q|$. Now it is clear that if $|\cdot|$ is a norm then $\varrho$ is a metric. Conversely, suppose $\varrho$ is a metric, $x, y \in X$ and $|x-y|=0$. First assume that $S_{0}$ is radial. Since $|x-y|=0$ there exist $p, q \in S_{0}, 0 \leqq c, d \leqq 1$ such that $x-y=c p-d q=p_{1}-q_{1}$ where $p_{1}, q_{1} \in S_{0}$. Then $\varrho\left(p_{1}, q_{1}\right)=\left|p_{1}-q_{1}\right|=0$ so $p_{1}=q_{1}$ and hence $x=y$. Next assume that $S_{0}$ is normalized. We first show that in this case $|p|=1$ for all $p \in S_{0}$. Indeed, if $p=c p_{1}-d q_{1}, p_{1}, q_{1} \in S_{0}, c, d \geqq 0$, then $(1+d)^{-1} p+d(1+d)^{-1} q_{1}$ $=c(1+d)^{-1} p_{1}$ and hence $c=1+\mathrm{d}$. Since $d \geqq 0, c \geqq 1$ and hence

$$
|p|=\inf \left\{\max (c, d): p=c p_{1}-d q_{1}, c, d \geqq 0, p_{1}, q_{1} \in S_{0}\right\} \geqq 1 \text {. }
$$

But $p=p-0 q$ so $|p|=1$. Now suppose $x-y=c p-d q, c, d \geqq 0, p, q \in S_{0}$. Then

$$
0=|x-y|=|c p-d q| \geqq|| c p|-| d q||=|c| p|-d| q||=|c-d|,
$$

so $c=d$. Hence $0=|x-y|=c|p-q|=c \varrho(p, q)$, if $c \neq 0$, then $\varrho(p, q)=0$, giving $p=q$ and $x=y$, if $c=0$ then again $x=y$.

In most examples $S_{0}$ is taken to be a positive convex set; that is if $p \in S_{0}$ then $\alpha p \notin S_{0}$ for all $\alpha<0$. Notice if $S_{0}$ is normalized, it is positive. Positivity has the advantage of making $K$ a cone. Indeed if $\alpha p=-\beta p_{1}$, $\alpha, \beta \geqq 0, p, p_{1} \in S_{0}$ then if $\alpha>0$ we have $p=-(\beta / \alpha) p_{1}$ so $p_{1} \in S_{0}$ and $-(\beta / \alpha) p_{1} \in S_{0}$ which is a contradiction. Hence $\alpha=0$ and $K \cap(-K)=\{0\}$. The relation between positivity and normalized is given in the following:

Corollary 3.3. $S_{0}$ is normalized if and only if it is positive and $|p|=1$ for all $p \in S_{0}$.

Proof. Necessity has been proved in the previous theorem. Conversely suppose $S_{0}$ is positive and $|p|=1$ for all $p \in S_{0}$. Then if $p \in S_{0}$ and $\alpha p \in S_{0}$ we have $1=|\alpha p|=|\alpha|$ so $\alpha= \pm 1$. Now $\alpha \neq-1$ by positivity. 
We now consider linear extensions of affine maps from $S_{0}$ to $X$. If $S_{0}$ is radial and $T: S_{0} \rightarrow S_{0}$ we say that $T$ is homogeneous if $T(\lambda p)=\lambda T p$ for all $p \in S_{0}$ and $0<\lambda<1$.

Theorem 3.4. Let $S_{0}$ be a normalized or a positive and radial convex set in a real vector space $V$ and let $|\cdot|$ be the induced seminorm on the subspace $X=K-K \subseteq V$. If $S_{0}$ is normalized (positive radial) and $T: S_{0} \rightarrow S_{0}$ is an affine (and homogeneous) map then $T$ has a unique linear extension $\hat{T}$ to $X$ and $\|\hat{T}\| \leqq 1$ (i.e., $|T x| \leqq|x|$ for all $x \in X$ ). If furthermore $T$ is a bijection then $\hat{T}$ is an isometry on $X$ (i.e., $|\hat{T} x|=|x|$ for all $x \in X)$.

Proof. Any $x \in X$ admits a representation $x=c p-d q, c, d \geqq 0$, $p, q \in S_{0}$. Define $\hat{T} x=c T p-d T q$. To show $\hat{T}$ is well-defined suppose also that $x=c_{1} p_{1}-d_{1} q_{1}, c_{1}, d_{1} \geqq 0, p_{1}, q_{1} \in S_{0}$. First suppose $S_{0}$ is normalized and $T$ is affine. Then since $c p-d q=c_{1} p_{1}-d_{1} q_{1}$ we have (notice $\left.c_{1}+d, c+d_{1}>0\right) c\left(c+d_{1}\right)^{-1} p+d_{1}\left(c+d_{1}\right)^{-1} q_{1}=\left(c_{1}+d\right)\left(c+d_{1}\right)^{-1}$ $\cdot\left(c_{1}\left(c_{1}+d\right)^{-1} p_{k}+d\left(c_{1}+d\right)^{-1} q\right)$ so $c_{1}+d=c+d_{1}$ and hence

$$
c\left(c+d_{1}\right)^{-1} T p+d_{1}\left(c+d_{1}\right)^{-1} T q_{1}=c_{1}\left(c+d_{1}\right)^{-1} T p_{1}+d\left(c_{1}+d\right)^{-1} q .
$$

It follows that $c T p=d T q=c_{1} T p_{1}-d_{1} T q_{1}$. Next suppose $S_{0}$ is positive, radial and $T$ is affine and homogeneous. By positivity we have $c_{1}+d$, $c+d_{1}>0$. Now either $c_{1}+d \leqq c+d_{1}$ or $c_{1}+d \geqq c+d$. For concreteness assume the former and apply the facts that $S_{0}$ is radial and $T$ is affine, homogeneous to obtain

$$
\begin{aligned}
& c\left(c+d_{1}\right)^{-1} T p+d_{1}\left(c+d_{1}\right)^{-1} T q_{1} \\
& \quad=\left(c_{1}+d\right)\left(c+d_{1}\right)^{-1}\left(c_{1}\left(c_{1}+d\right)^{-1} T p_{1}+d\left(c_{1}+d\right)^{-1} T q\right) .
\end{aligned}
$$

Again it follows that $c T p-d T q=c_{1} T p_{1}-d T q_{1}$. Thus $\hat{T}$ is well-defined and it is easy to show that $\hat{T}$ is a linear operator on $X$. To show $\hat{T}$ is a contraction we have for $x \in X$

$$
\begin{aligned}
|\hat{T} x| & =\inf \left\{\max (c, d): \hat{T} x=c p-d q, c, d \geqq 0, p, q \in S_{0}\right\} \\
& \leqq \inf \left\{\max (c, d): x=c p-d q, c, d \geqq 0, p, q \in S_{0}\right\}=|x|
\end{aligned}
$$

Corollary 3.5. Let $S_{0}, X,|\cdot|$ be as in the previous theorem. If $T$ is a linear operator on $X$ that leaves $S_{0}$ invariant then $T$ is a contraction (i.e., $|T x| \leqq|x|$ for all $x \in X$ ). If furthermore Trestricted to $S_{0}$ is a bijection then $T$ is an isometry (i.e., $|T x|=|x|$ for all $x \in X$.)

Theorem 3.6. Let $S_{0}$ be a normalized or radial convex set in a real vector space $V$ and let $X$ be the generated subspace. Let $\varrho$ be the intrinsic semi-metric on $S_{0}$ and $|\cdot|$ the induced seminorm on $X$. If $\left(S_{0}, \varrho\right)$ is complete then so is $(X,|\cdot|)$. 
Proof. First note that for $p \in S_{0}$, since $p=1 p$ we have $|p| \leqq 1$. Assuming $\left(S_{0}, \varrho\right)$ is complete, let $x_{n}$ be a Cauchy sequence in $X$. We may assume that $\left|x_{n+1}-x_{n}\right|<2^{-n}$ for $n=1,2, \ldots$. Now we can write $x_{n+1}-x_{n}=c_{n} p_{n}-d_{n} q_{n}$ where $0 \leqq c_{n}, d_{n}<2^{-n}, p_{n}, q_{n} \in S_{0}$. We can assume $c_{1}, d_{1}>0$. Let

Now

$$
a_{n}=\sum_{i=1}^{n} c_{i}, \quad b_{n}=\sum_{i=1}^{n} d_{i} .
$$

and

$$
\begin{array}{r}
\left\{\sum_{i=1}^{n} a_{n}^{-1} c_{i} p_{i}, i=1,2, \ldots\right\} \\
\left\{\sum_{i=1}^{n} b_{n}^{-1} d_{i} q_{i}, i=1,2, \ldots\right\}
\end{array}
$$

are Cauchy sequences in $S_{0}$. Indeed, it is clear that $\left\{a_{n}\right\}$ is a Cauchy sequence and we have

$$
\begin{aligned}
\varrho\left(\sum_{i=1}^{n+k} a_{n+k}^{-1} c_{i} p_{i}\right. & \left., \sum_{i=1}^{n} a_{n}^{-1} c_{i} p_{i}\right) \\
= & \left|\left[a_{n+1}^{-1}-a_{n}^{-1}\right] \sum_{i=1}^{n} c_{i} p_{i}+\sum_{i=n+1}^{n+k} a_{n+k}^{-1} c_{i} p_{i}\right| \\
\leqq & \left(a_{n}^{-1}-a_{n+1}^{-1}\right) \sum_{i=1}^{n} c_{i}+a_{n+k}^{-1} \sum_{i=n+1}^{n+k} c_{i} \\
= & 1-a_{n} a_{n+k}^{-1}\left(a_{n+k}-a_{n}\right)=2\left(1-a_{n} a_{n+k}^{-1}\right),
\end{aligned}
$$

where the last term approaches zero as $n, k \rightarrow \infty$. Thus there are elements $p, q \in S_{0}$ such that $\sum_{i=1}^{n} a_{n}^{-1} c_{i} p_{i} \rightarrow p$ and $\sum_{i=1}^{n} b_{n}^{-1} d_{i} q_{i} \rightarrow q$. Suppose $a_{n} \rightarrow a$ and $b_{n} \rightarrow b$; we claim that $x_{n} \rightarrow x_{1}+a p-b q$. Indeed,

$$
\begin{aligned}
\left|x_{n+1}-x_{1}-a p+b q\right| & =\left|x_{n+1}-x_{n}+x_{n}-x_{n-1}+\cdots+x_{2}-x_{1}-a p+b q\right| \\
& \leqq|a|\left[\sum_{i=1}^{n} a^{-1} c_{i} p_{i}-p|+| b|| \sum_{i=1}^{n} b^{-1} d_{i} q_{i}-q \mid\right. \\
& \leqq|a|\left[\left|\sum_{i=1}^{n}\left(a^{-1} c_{i}-a_{n}^{-1} c_{i}\right) p_{i}\right|+\left|\sum_{i=1}^{n} a_{n}^{-1} c_{i} p_{i}-p\right|\right] \\
& +|b|\left[\left|\sum\left(b^{-1} d_{i}-b_{n}^{-1} d_{i}\right) q_{i}\right|+\left|\sum_{i=1}^{n} b_{n}^{-1} d_{i} q_{i}-q\right|\right] \\
& \leqq|a|\left[1-a^{-1} a_{n}+\left|\sum_{i=1}^{n} a_{n}^{-1} c_{i} p_{i}-p\right|\right] \\
& +|b|\left[1-b^{-1} b_{n}+\left|\sum_{i=1}^{n} b_{n}^{-1} d_{i} q_{i}-q\right|\right]
\end{aligned}
$$

$\rightarrow 0$ as $n \rightarrow \infty$.

We next consider the geometry of $K$ relative to $|\cdot|$. 
Lemma 3.7. Let $S_{0}$ be a normalized or radial convex set in $V, X$ the generated subspace and $|\cdot|$ the induced seminorm on $X$. (1) If $x, y \in X$ and $x-y \in K$, then $|x| \geqq|y|$. (2) Let $\gamma>1$. For every $x \in X$ there exists $x_{1}, x_{2} \in K$ such that $x=x_{1}-x_{2}$ and $\left|x_{i}\right| \leqq \gamma|x|, i=1,2$.

Proof. (1) Since $x-y \in K$ there is a $c_{0} \geqq 0$ and $p_{0} \in S_{0}$ such that $x-y=c_{0} p_{0}$. We may assume $c_{0}>0$. If $y=c p-d q, c, d \geqq 0, p, q \in S_{0}$ then $x=y+c_{0} p_{0}=\left(c_{0}+c\right)\left[c_{0}\left(c+c_{0}\right)^{-1} p_{0}+c\left(c+c_{0}\right)^{-1} p\right]-d q$ and hence $|x| \geqq|y|$. (2) For $x \in X$, by definition of $|x|$ there exist $c, d \leqq \gamma|x|$, $p, q \in S_{0}$ such that $x=c p-d q$. Letting $x_{0}=c p, x_{2}=d q$ we have $x_{1}, x_{2} \in K, x=x_{1}-x_{2}$ and $\left|x_{1}\right|=|c p| \leqq c \leqq \gamma|x|$ and $\left|x_{2}\right| \leqq \gamma|x|$.

If $K$ is a cone (e. g. if $S_{0}$ is positive) it follows from Lemma 3.7 that $K$ is a normal strict $b$-cone in $X[12]$.

Another norm on $X$ that appears in the literature (we consider this norm in the next section) is the natural seminorm

$$
|x|_{1}=\inf \left\{c+d: x=c p-d q ; c, d \geqq 0, p, q \in S_{0}\right\} .
$$

It is clear that $|x| \leqq|x|_{1} \leqq 2|x|$. The natural seminorm reduces to the natural semimetric $\varrho_{1}(p, q)$ which is twice the intrinsic semimetric. Indeed from the proof of Theorem 3.2 we have for $p, q \in S_{0}$

$$
\begin{aligned}
\varrho_{1}(p, q) & =|p-q|_{1}=\inf \left\{c+d: p-q=c p_{1}-d q_{1}, c, d \geqq 0, p_{1}, q_{1} \in S_{0}\right\} \\
& =\inf \left\{2 c: p-q=c\left(p_{1}-q_{1}\right), c \geqq 0, p_{1}, q_{1} \in S_{0}\right\} \\
& =2|p-q|=2 \varrho(p, q) .
\end{aligned}
$$

Let us now compare our theory with the usual Hilbert space theory. In this case the normalized states $\mathscr{D}$ are the convex set of density operators; that is, the set of positive trace class operators with trace one. $\mathscr{D}$ is a base for the cone $K$ of positive trace class operators which forms the set of states and $K$ generates the real vector space $X=K-K$ of generalized states consisting of the self-adjoint trace class operators. In their latest study of scattering theory, Jauch, Misra and Gibson [8] use the trace norm $|\cdot|_{1}$ on $X$; that is, for $x \in X,|x|_{1}=\Sigma\left|\lambda_{i}\right|$ where $\lambda_{i}$ are the repeated eigenvalues of $x$. Now $|\cdot|_{1}$ reduces to the trace metric $\varrho_{1}$ on $\mathscr{D}$. We now compare the intrinsic metric $\varrho$ and the induced norm $|\cdot|$ to $\varrho_{1}$ and $|\cdot|_{1}$ respectively.

Lemma 3.8. Let $x \in X$ and let $\left\{\lambda_{i}\right\},\left\{-\mu_{i}\right\}$ be the repeated positive eigenvalues and negative eigenvalues respectively of $x$. Then $x=x_{1}-x_{2}$, where $x_{1}, x_{2} \in K, \operatorname{tr} x_{1}=\Sigma \lambda_{i}, \operatorname{tr} x_{2}=\Sigma \mu_{i}$ and if $x=y_{1}-y_{2}, y_{1}, y_{2} \in K$ then $\operatorname{tr} y_{1} \geqq \operatorname{tr} x_{1}, \operatorname{tr} y_{2} \geqq \operatorname{tr} x_{2}$.

Proof. Let $f^{+}(\lambda)=\lambda$ for $\lambda \geqq 0$ and $f^{+}(\lambda)=0$ for $\lambda \leqq 0$. Let $f^{-}(\lambda)=-\lambda$ for $\lambda \leqq 0$ and $f^{-}(\lambda)=0$ for $\lambda \geqq 0$. Then by the spectral theorem

$$
\begin{aligned}
x & =\int \lambda P^{x}(d \lambda)=\int_{\{\lambda>0\}} \lambda P^{x}(d \lambda)+\int_{\{\lambda<0\}} \lambda P^{x}(d \lambda)=f^{+}(x)-f^{-}(x) \\
& \equiv x_{1}-x_{2} .
\end{aligned}
$$


It is clear that the spectra $\sigma\left(x_{1}\right)=\left\{\lambda_{i}\right\}, \sigma\left(x_{2}\right)=\left\{\mu_{i}\right\}$. Now suppose $x=y_{1}=y_{2}, y_{1}, y_{2} \in K$. If $\phi$ is an eigenvector of $x$ corresponding to a positive eigenvalue then

$$
\begin{aligned}
\left\langle y_{1} \phi, \phi\right\rangle & =\langle x \phi, \phi\rangle+\left\langle y_{2} \phi, \phi\right\rangle=\left\langle x_{1} \phi, \phi\right\rangle+\left\langle y_{2} \phi, \phi\right\rangle \\
& \geqq\left\langle x_{1} \phi, \phi\right\rangle .
\end{aligned}
$$

If $\phi$ is an eigenvector of $x$ corresponding to a negative eigenvalue then $\left\langle y_{1} \phi, \phi\right\rangle \geqq 0=\left\langle x_{1} \phi, \phi\right\rangle$. Computing the traces using the eigenvectors of $x$ we have $\operatorname{tr} y_{1} \geqq \operatorname{tr} x_{1}$ and similarly $\operatorname{tr} y_{2} \geqq \operatorname{tr} x_{2}$.

Corollary 3.9. If $x \in X$ then

$$
|x|_{1}=\inf \left\{c+d: x=c p_{1}-d q_{1}, c, d \geqq 0, p_{1}, q_{1} \in \mathscr{D}\right\} .
$$

Proof. Applying Lemma 3.8,

so

$$
x=x_{1}-x_{2}=\left(\operatorname{tr} x_{1}\right)\left[\left(\operatorname{tr} x_{1}\right)^{-1} x_{1}\right]-\left(\operatorname{tr} x_{2}\right)\left[\left(\operatorname{tr} x_{2}\right)^{-1} x_{2}\right]
$$

$$
\left.x\right|_{1}=\operatorname{tr} x_{1}+\operatorname{tr} x_{2}=\inf \left\{c+d: x=c p_{1}-d q_{1}, c, d \geqq 0, p_{1}, q_{1} \in \mathscr{D}\right\} .
$$

This completes the proof.

It follows from Lemma 3.8 that $|x|=\max \left(\left|x_{1}\right|_{1},\left|x_{2}\right|_{1}\right)=\max \left(\Sigma \lambda_{i}, \Sigma \mu_{i}\right)$. Applying Corollary 3.9 we see that $|\cdot|_{1}$ is the natural norm considered in the previous section. It follows from the work of that section that $|x| \leqq|x|_{1} \leqq 2|x|$ so $|\cdot|$ and $|\cdot|_{1}$ are equivalent and that $\varrho_{1}(p, q)=2 \varrho(p, q)$ for all $p, q \in \mathscr{D}$. In particular if $\left\{\lambda_{i}\right\}$ and $\left\{-\mu_{i}\right\}$ are the repeated positive and negative eigenvalues of $p-q$ we have $\Sigma \lambda_{i}+\Sigma \mu_{i}=2 \max \left(\Sigma \lambda_{i}, \Sigma \mu_{i}\right)$. Thus $\Sigma \lambda_{i}=\Sigma \mu_{i}$ which can also be easily derived from other means.

Jauch, Misra and Gibson [8] have shown that if $p, q \in \mathscr{D}$ are pure states corresponding to unit vectors $\phi, \psi$ then $\varrho_{1}(p, q)=2\left(1-|\langle\phi, \psi\rangle|^{2}\right)^{1 / 2}$. It follows that $\varrho(p, q)^{2}=1-|\langle\phi, \psi\rangle|^{2}$.

\section{Operational Quantum Mechanics}

Returning to our general convex structure of states $S$, suppose there are enough observables to distinguish between states or equivalently that $S^{*}$ is total. It then follows from Theorem 2.2 that $S_{1}$ is isomorphic to a convex set in a real linear space $V$. Then $S$ is a cone with base $S_{1}$ and $X=S-S$ is a subspace of $V$ generated by $S$. Let us make the reasonable assumption that the intrinsic semimetric $\varrho$ is a metric and form the completion $\tilde{S}_{1}$ of $S_{1}$. Then $\tilde{S}_{1}$ is a base for a cone $\tilde{S}$ which generates the linear space $\tilde{X}=\tilde{S}-\tilde{S}$. If $|\cdot|$ is the intrinsic norm on $\tilde{X}$ then $\tilde{S}_{1}$ is normalized and hence by Theorem $3.6(\tilde{X},|\cdot|)$ is a Banach space with closed generating cone $\tilde{S}$. Defining $\tau$ as above we see that $\tau(x)=|x|$ for every $x \in \tilde{S}$ and the triple $(\tilde{X}, \tilde{S}, \tau)$ becomes a complete base normed space (or state space), 
the basic framework for the operational quantum mechanics of Davies and Lewis. The norm $|\cdot|$ is equivalent to the natural norm $|\cdot|_{1}$ used by Davies and Lewis and applying Theorem 3.4 one can give definitions of observables, instruments, joint distributions, etc. on $S$ which reduce to theirs. We thus see that in the case of a separating set of observables our framework reduces to that of Davies and Lewis and hence gives a simple, axiomatic motivation for their theory.

Mielnik gives convincing arguments for describing quantal situations in terms of a basic mathematical structure called a quantum system $(e, D, T, B)$. In this structure, $D$ is a linear subspace of the algebraic dual $X^{*}$ of a real linear space $X$. Elements of $D$ are called detectors. We give $X$ the topology induced by $D$ and assuming $D$ separates points of $X$ this makes $X$ into a Hausdorff locally convex space. $B$ is a generating closed cone for $X$ whose elements are called beams. Denoting the set of continuous linear operators on $X$ by $\mathscr{L}(X), T$ is a positive algebra in $\mathscr{L}(X)$ such that $T(B) \cong B$. That is, $T$ is closed under composition, addition, and multiplication by nonnegative scalars. $T$ is called the algebra of transmitters. Finally $e \in D$ is a distinguished detector called the standard quantum detector or quantum scale and satisfies (1) $e x \geqq 0$ for all $x \in B$, (2) if $x \in B$ and $e x=0$ then $x=0$. The set $S=\{x \in B: e x=1\}$ is the figure of states or the statistical figure. Although the Mielnik formalism is similar to that of Davies and Lewis, the former stresses convex set methods while the latter stresses the operational methods of probability theory.

It is clear that a Mielnik quantum system is a special case of our convex structure. Conversely, if $\left(S_{1},\langle\cdot, \cdot, \cdot\rangle\right)$ is a convex structure then $S_{1}$ gives the statistical figure, $S=\left\{\alpha S_{1}: \alpha \geqq 0\right\}$ is the set of beams, a subspace $D \cong S^{*}$ gives the set of detectors, $A f(S)$ the set of transmitters, and $\tau$ the quantum scale. If it is assumed that $S^{*}$ (or as Mielnik assumes, $D$ ) is total over $S$ then applying Theorems 2.2 and 3.4 our structure reduces to a Mielnik quantum system. However, if $S^{*}$ is not total over $S$ then we get a non-linear generalization of Mielnik's theory.

\section{References}

1. Cornette, W., Ph.D.: Dissertation, University of Denver (in preparation).

2. Davies, E. B., Lewis, J. T.: An operational approach to quantum probability. Commun. math. Phys. 17, 239-260 (1970).

3. Edwards, C. M.: Classes of operations in quantum theory. Commun. math. Phys. 20, 26-56 (1971).

4. Gudder,S.: Axiomatic quantum mechanics and generalized probability theory in A. Bharucha-Reid (editor), Probabilistic Methods in Applied Mathematics, Vol. II. New York: Academic Press 1970. 
5. Gunson.J.: Structure of quantum mechanics. Commun. math. Phys. 6, 262-285 (1967).

6. Haag, R., Kastler, D.: An algebraic approach to quantum field theory. J. Math. Phys. 5, 848-861 (1964).

7. Jauch, J.: Foundations of quantum mechanics. Reading, Mass. : Addison-Wesley 1968.

8. Jauch, J., Misra, B., Gibson, A.: On the asymptotic condition of scattering theory. Helv. Phys. Acta 41, 513-527 (1968).

9. Mielnik, B.: Geometry of quantum states. Commun. math. Phys. 9, 55-80 (1968).

10. Mielnik, B.: Theory of filters. Commun. math. Phys. 15, 1-46 (1969).

11. Neumann, H.: Classical systems and observables in quantum mechanics. Commun. math. Phys. 23, 100-116 (1971).

12. Peressini, A.: Ordered Topological Vector Spaces. New York: Harper and Row 1967.

13. Stolz, P.: Attempt of an axiomatic foundation of quantum mechanics and more general theories, VI. Commun. math. Phys. 23, 117-126 (1971).

14. Segal, I.: Mathematical problems of relativistic physics. American Math. Soc. Lectures in Applied Mathematics. Providence, R.I. (1963).

15. Varadarajan, V.: Geometry of Quantum Theory, Vol. I. Princeton, N.J.: Van Nostrand 1968.

\author{
S. Gudder \\ Department of Mathematics \\ University of Denver \\ Denver Colorado 80210, USA
}

\title{
Erratum
}

\section{Non-Existence of Axially Symmetric Massive Scalar Fields}

\author{
A. R. Roy and J. R. Rao \\ Commun. math. Phys. 27, 162-166 (1972)
}

Page 165: 4th line onwards from the top reads as follows:

Which in view of (3.4) implies

$$
-\mu^{2} V^{2}+g^{11} g^{44}\left(F_{14}\right)^{2}-g^{22} g^{33}\left(F_{23}\right)^{2}=0 .
$$

Since $g^{11}, g^{22}, g^{33}$ are all negative and $g^{44}$ is positive, (3.5) will hold iff

$$
\mu=0, \quad F_{14}=0, \quad F_{23}=0 .
$$

Hence, there cannot exist any solution for the coupled electromagnetic and massive scalar fields for the metric (2.1).

The rest of the calculations are unnecessary and may, therefore, be ignored. 